\title{
QUALIDADE DE VIDA E RESILIÊNCIA EM PACIENTES PÓS-OPERATÓRIO DE CIRURGIA CARDÍACA
}

\author{
Davisson Gonçalves Giaretta ${ }^{\mathrm{a}^{*}}$, Evelyn Soledad Reyes Vigueras ${ }^{\mathrm{b}^{*}}$, \\ Patricia Pereira Ruschel ${ }^{c^{*}}$, Camila Souza $^{\mathrm{d}^{*}}$
}

davissongi@hotmail.com ${ }^{\mathrm{a}}$, evelynvigueras@hotmail.com ${ }^{\mathrm{b}}$,patriciapruschel@gmail.com ${ }^{\mathrm{c}}$,psico.camilasouza@gmail.com ${ }^{\mathrm{d}}$ Faculdade de Medicina da Pontifícia Universidade Católica do Rio Grande do Sul*

\section{RESUMO}

Introdução: Em âmbito mundial, as doenças crônicas não transmissíveis são responsáveis pelas principais causas de morte e incapacidade. Entre essas, encontram-se as de maior importância para a saúde pública, as doenças cardiovasculares. Objetivo: Tendo em vista que a capacidade de resiliência e a Qualidade de Vida são construtos relevantes a serem considerados em pacientes internados em ambiente hospitalar, o presente estudo objetiva verificar como se apresentam esses conceitos em indivíduos que realizaram cirurgia de revascularização do miocárdio e/ou cirurgia de válvula em uma instituição hospitalar. Metodologia: Para tanto, foi realizada uma pesquisa transversal na qual foram aplicados um questionário sociodemográfico, $\mathrm{o}$ questionário de qualidade de vida SF-12 e uma escala de resiliência. Participaram desse estudo 37 pacientes, maioria do sexo masculino, submetidos à primeira intervenção cirúrgica. Resultados: Observou-se uma média de 37,3 pontos ( $\mathrm{dp} \pm 4,4)$ e 43,3 (dp $\pm 7,6)$ nos componentes físico e mental da escala de Qualidade de Vida SF-12, respectivamente. Já a escala de Resiliência aplicada revelou média de 131 (dp \pm 31$)$. Conclusão: Verificou-se uma correlação direta fraca entre o componente físico da qualidade de vida com o escore de resiliência $(r=0,386, p=0,02)$, o que sugere tendência positiva entre tais conceitos.

Palavras-chave: Cirurgia Torácica, Qualidade de Vida, Resiliência Psicológica

\begin{abstract}
Introduction: Worldwide, chronic noncommunicable diseases account for the leading causes of death and disability. Among these are those of major importance for public health, cardiovascular diseases. Objective: Considering that resilience capacity and Quality of Life are relevant constructs to be considered in hospitalized patients, the present study aims to verify how these concepts are found in people who underwent coronary artery bypass and/or valve surgery in a hospital. Methods:For that, a cross-sectional study was designed in which a sociodemographic questionnaire, the SF-12 quality of life questionnaire and a resilience scale were applied. Results: A total of 37 patients, mostly male, underwent the first surgical intervention. An average of 37.3 points $(\mathrm{dp} \pm 4.4)$ and $43.3(\mathrm{dp} \pm 7.6)$ were observed in the physical and mental components of the SF-12 Quality of Life scale, respectively. The Resilience scale revealed a mean of 131 ( $\mathrm{dp} \pm 31$ ). Conclusion: There was a weak direct correlation between the physical component of quality of life and the resilience score $(r=0.386, p=0.02)$, suggesting a positive trend among these concepts.
\end{abstract}

Key words: Thoracic Surgery, Quality of Life, Psychological Resilience 


\section{INTRODUÇÃO}

No contexto mundial, as principais causas de morte e incapacidade estão relacionadas às doenças crônicas não transmissíveis. Dentre tais doenças, as de maior importância para a saúde pública encontram-se, as cardiovasculares, o câncer e o diabetes mellitus ${ }^{1}$. Segundo os autores, na esfera das doenças cardiovasculares, sobressaem-se a doença isquêmica cardíaca, as doenças cerebrovascular e a hipertensão arterial sistêmica (HAS). Além disso, vale ressaltar que dentre as patologias cardíacas encontram-se as valvopatias, que consistem em afecções que acometem as válvulas do coração, que também são foco de atenção no que se refere à saúde cardíaca ${ }^{2}$.

Para alcançar uma compreensão mais ampla dos efeitos da cardiopatia na vida do sujeito, faz-se necessário versar brevemente acerca da qualidade de vida e da capacidade de resiliência do sujeito. Esses conceitos são bastante utilizados no campo da saúde. A qualidade de vida se refere a uma noção eminentemente humana, que tem sido aproximada ao grau de satisfação encontrado na vida familiar, amorosa, social e ambiental e à própria estética existencial. Pressupõe a capacidade de efetuar uma síntese cultural de todos os elementos que determinada sociedade considera seu padrão de conforto e bem-estar ${ }^{3}$.

A Organização Mundial da Saúde (OMS) conceitua Qualidade de Vida como a percepção do indivíduo de sua posição na vida no contexto da cultura e sistema de valores nos quais ele vive e em relação aos seus objetivos, expectativas, padrões e preocupações. É um amplo conceito de classificação, afetado de modo complexo pela saúde física do indivíduo, estado psicológico, relações sociais, nível de independência e pelas relações com as características mais relevantes do seu meio ambiente ${ }^{4}$.

Entende-se, também, que a Qualidade de Vida é uma noção eminentemente humana, que tem sido aproximada ao grau de satisfação encontrada na vida familiar, amorosa, social e ambiental, bem como, à própria estética existencial. Assim, é possível observar a abrangência de significados do termo, direcionando-o para todos os elementos que são considerados como padrão de bem-estar e conforto por determinada sociedade, inclusive abordando a satisfação do sujeito consigo mesmo ${ }^{5}$.
Já a resiliência caracteriza-se pela capacidade de o ser humano responder às demandas da vida cotidiana de forma positiva, apesar das adversidades que enfrenta ao longo de seu ciclo vital de desenvolvimento. Desse modo, resiliência alude à combinação entre os atributos do indivíduo e de seu ambiente familiar, social e cultural ${ }^{6}$.

Esse construto consiste em um conceito evolutivo e de saúde que caracteriza os processos dinâmicos que facilitam a organização e a integração da experiência em modos de funcionamento adaptativos. Este é um conceito que reafirma a capacidade do ser humano em superar situações potencialmente traumáticas, dando a possibilidade de um desenvolvimento adaptativo mesmo em cenários conflitivos ${ }^{7}$. A resiliência, portanto, não nasce com o sujeito, mas sim é adquirida ao longo de seu desenvolvimento ${ }^{8}$.

Para compreender a resiliência é fundamental conhecer como as características protetoras se desenvolveram e de que modo modificaram o percurso pessoal do indivíduo. Assim, a vulnerabilidade e os mecanismos protetores podem ser definidos como a capacidade que o indivíduo tem de modificar respostas frente a situações de risco ${ }^{9}$. O conceito de vulnerabilidade dá conta de uma intensificação da reação frente a estímulos que, em situações normais conduziriam a uma desadaptação. Os mecanismos protetores, ao contrário, seriam fatores de atenuação que atuam diante do estímulo9. Os fatores de proteção serão aqueles que, numa trajetória de risco, acabam modificando o curso da vida do sujeito de forma mais adaptativa, estando diretamente associados ao desenvolvimento saudável ${ }^{10}$. Para os autores, os fatores protetivos podem relacionar-se a aspectos individuais, como autoestima, autocontrole, autonomia, orientação social positiva; a aspectos familiares, como coesão e estabilidade; e a fatores do meio ambiente no qual o indivíduo está inserido, como relacionamento saudável com colegas e amigos

As bases da resiliência apresentam-se tanto como constitucionais quanto ambientais, o que significa que não existiria, portanto, uma quantidade fixa de processos de resiliência em um indivíduo, e sim o modo de enfrentar crises é que varia de acordo com as circunstâncias. A resiliência não é considerada uma 
capacidade inata ou herdada, mas se dá a partir da interação dinâmica existente entre as características individuais e o contexto social ${ }^{11}$. Desse modo, seria impróprio considerar uma pessoa como resiliente, em termos diagnósticos, porque o termo resiliência é a descrição de um modelo geral de personalidade. Seria, então, mais apropriado dizer que uma pessoa possui características de resiliência em sua personalidade, que podem ser transitórias e específicas a alguns contextos ${ }^{7}$.

No cenário hospitalar, mais especificamente no âmbito da cirurgia cardíaca, sabe-se que tal procedimento invasivo é considerado um evento adverso, gerador de estresse emocional. $\mathrm{O}$ enfrentamento dessa situação consiste na busca por respostas adaptativas e no manejo das experiências, estados emocionais e comportamentos causados pelo estresse ${ }^{12}$.

Tendo em vista o cenário, muitas vezes adverso, da cirurgia cardíaca, é inegável a existência de efeitos na subjetividade do sujeito o qual se submeterá a essa intervenção invasiva. Esse efeito, num primeiro momento, pode ser vivenciado como um impacto emocional. Tal fato está associado com a capacidade (de resiliência) de o paciente enfrentar esse evento estressor, repercutindo, assim, em sua qualidade de vida. Desse modo, justifica-se a extrema relevância de investigar e conhecer a capacidade de resiliência e a qualidade de vida dessa população a fim de refletir e de elaborar ações que visem o cuidado dessa população. Essas ações de cuidado referem-se tanto ao período pós-operatório, bem como ao momento anterior à cirurgia, em que as ações assumem um caráter preventivo e de promoção de saúde.

Dessa forma, o presente estudo visa avaliar a capacidade de resiliência e a qualidade de vida de pacientes adultos pós-operatório de cirurgia cardíaca. Objetiva-se, também, caracterizar a população estudada a partir dos dados sociodemográficos; conhecer os escores de Qualidade de Vida da população alvo, bem como, conferir a capacidade de resiliência dos pacientes pós-operatório de cirurgia cardíaca. Por fim, busca-se verificar a correlação entre qualidade de vida e resiliência.

\section{MÉTODO}

O presente artigo retrata um estudo transversal que investigou e comparou as variáveis qualidade de vida e resiliência. Participaram dessa pesquisa 37 pacientes adultos (a partir de 18 anos), os quais foram submetidos à intervenção cirúrgica cardíaca em um hospital na região metropolitana de Porto Alegre. Os participantes realizaram cirurgia de revascularização do miocárdio ou cirurgia de válvula. Ressalta-se que esse estudo foi submetido e aprovado pelo Comitê de Ética em Pesquisa da referida instituição, com Número de Parecer 879.281, atendendo aos trâmites éticos. Os participantes assinaram o Termo de Consentimento Livre e Esclarecido (TCLE). Foram excluídos da pesquisa os pacientes que apresentaram alguma impossibilidade de responder aos questionários.

Para a coleta dos dados utilizaram-se os seguintes instrumentos:

- Questionário sociodemográfico: desenvolvido para esta pesquisa a fim de coletar dados sociais e demográficos visando a caracterização da amostra;

- Questionário de Avaliação de Qualidade de Vida SF-12: composto por doze itens; avalia dois diferentes componentes da qualidade de vida - físico e mental -, considerando a percepção do indivíduo em relação aos aspectos de sua saúde nas quatro últimas semanas;

- Escala de Resiliência: possui 25 itens descritos de forma positiva divididos em três fatores: fator 1 (transmite a ideia de resolução de ações e valores); fator 2 (indica independência e determinação) e fator 3 (infere sobre autoconfiança e capacidade de adaptação).

Os questionários e escalas foram aplicados após a realização de cirurgia cardíaca, durante a internação na enfermaria. Em um primeiro momento, os dados coletados foram armazenados em uma planilha Excel e posteriormente analisados no programa SPSS. No que tange à análise dos dados, as 
variáveis qualitativas foram descritas através da frequência absoluta e frequência relativa e as variáveis quantitativas, através de média e desvio padrão. Para correlacionar os escores de qualidade de vida e resiliência foi utilizada a correlação de Pearson.

\section{RESULTADOS}

A partir dos dados coletados e analisados observa-se que a maior parte da amos- tra compreende sujeitos do sexo masculino, correspondendo a 22 participantes (59,5\%). A idade variou de 19 a 87 anos, com média de 56,54 anos ( $d p \pm 10,08)$. A situação laboral atual foi predominantemente inativo $(64,9 \%)$, ou seja, a maioria dos participantes não estava trabalhando no momento da realização desse estudo. Quanto à intervenção cirúrgica, 33 sujeitos $(89,2 \%)$ submeteram-se à primeira cirurgia cardíaca. A Tabela 1 retrata a caracterização da amostra.

Tabela 1 - Caracterização da amostra

\begin{tabular}{cc}
\hline Caracterização da Amostra & N $=\mathbf{3 7}$ \\
\hline Idade & $56,54 \pm 10,08$ \\
\hline Anos de estudo & $7,86 \pm 3,13$ \\
\hline Sexo (masculino) & $22(59,5)$ \\
\hline Estado civil (casado) & $24(64,9)$ \\
\hline Atividade laboral (inativo) & $24(64,9)$ \\
\hline $1^{\text {a }}$ cirurgia cardíaca & $33(89,2)$ \\
\hline Cirurgia de Revascularização do Miocárdio (CRM) & $22(59,5)$ \\
\hline CRM e Cirurgia de Válvula & $14(37,8)$ \\
\hline Recebeu explicação sobre a cir. da equipe & $1(2,7)$ \\
\hline Compreendeu as explicação (auto referido) & $37(100)$ \\
\hline
\end{tabular}

média $\pm \mathrm{dp}, \mathrm{n}(\%)$

Já no que tange aos fatores de risco, constatou-se presença de Hipertensão Arterial Sistêmica $(48,6 \%)$, seguido de Diabetes $(37,8 \%)$ e estresse $(37,8 \%)$, conforme ilustra a tabela 2. Vale destacar que essas informações foram referidas pelo próprio participante durante o preenchimento do questionário sociodemográfico.

Tabela 2 - Fatores de risco

\begin{tabular}{ll}
\hline Fator de Risco & $\mathbf{N}=\mathbf{3 7} \quad \mathbf{n}(\mathbf{\%})$ \\
\hline HAS & $18(48,6)$ \\
\hline Diabetes & $14(37,8)$ \\
\hline Estresse & $14(37,8)$ \\
\hline
\end{tabular}




\begin{tabular}{ll}
\hline Sedentarismo & $13(35,1)$ \\
\hline Obesidade & $6(16,2)$ \\
\hline Tabagismo & $5(13,5)$ \\
\hline $\mathrm{n}(\%)$ &
\end{tabular}

Em relação ao questionário SF-12, o qual avalia qualidade de vida nos componentes físico e mental, constatou-se um escore médio de 37,3 $(\mathrm{dp} \pm 4,4)$ no primeiro componente. E uma média de 43,3 (dp $\pm 7,6)$ no componente mental. Já no que diz respeito à escala de Resiliência, o escore médio foi de 131 pontos $(\mathrm{dp} \pm 31)$.
Ao comparar os resultados do questionário SF-12 com a Escala de Resiliência, observa-se uma correlação direta fraca entre o componente físico do instrumento SF-12 e a escala de Resiliência, demonstrado pelo coeficiente de correlação de Pearson $(r=0,386, p<0,05)$, conforme evidencia a tabela 3 .

Tabela 3 - Correlação de Pearson entre Qualidade de Vida e Resiliência

\begin{tabular}{llll}
\hline & \multicolumn{3}{c}{ Resiliência } \\
\hline \multirow{3}{*}{ SF-12 } & $\begin{array}{l}\text { Component e } \\
\text { Físico }\end{array}$ & $\mathrm{r}=0,386$ & $\mathrm{p}=0,02$ \\
\cline { 2 - 4 } & $\begin{array}{l}\text { Component e } \\
\text { Mental }\end{array}$ & $\mathrm{r}=0,196$ & $\mathrm{p}=0,25$ \\
\hline
\end{tabular}

A correlação evidenciada por esse estudo indica uma tendência positiva entre os construtos Resiliência e Qualidade de Vida. Ou seja, quando observada, na amostra, uma elevada capacidade de resiliência, a qualidade de vida em relação ao componente físico tende a ser, também, elevada.

\section{DISCUSSÃO}

Segundo o Ministério da Saúde, as doenças isquêmicas do coração correspondem ao segundo lugar entre as causas de morte no Brasil. Dentre o sexo masculino, elas são consideradas a maior causa de óbito ${ }^{13}$.

Atualmente, com a medicina baseada em evidência, é possível compreender os mecanismos das doenças cardiovasculares. Mecanismos esses que vão desde a fisiopatologia e diagnóstico, até o prognóstico e terapêutica. O que ainda carece de uma abordagem mais eficiente, principalmetne no que se refere a políticas de saúde pública permanentes, é a prevenção e o controle dos fatores de risco $^{14}$. No que tange aos fatores de risco para doença cardiovascular, Gus, Fischmann e Medina ${ }^{15}$ realizaram pesquisa observacional com delineamento transversal a fim de conhecer a prevalência dos principais fatores para doença arterial coronariana no estado do Rio Grande do Sul. Os autores identificaram o sedentarismo como o fator mais prevalente $(71,3 \%)$. Também observaram elevada prevalência de sobrepesso/obesidade $(54,7 \%)$; sexo masculino (48,2\%); hipertensão arterial sistêmica $(46 \%)$ e tabagismo $(33,9 \%)^{15}$.

Esses dados corroboram os achados da presente pesquisa, tendo em vista que $48,6 \%$ da amostra desse estudo apresentou hipertensão arterial sistêmica (HAS); 35,1\% declararam-se sedentários e 59,5\% da amostra é constituída por homens. Constata-se, assim, que o controle das variáveis HAS e sedentarismo é considerado importante fator para a prevenção de doença arterial coronariana.

A Qualidade de Vida consiste em um constructo amplo, o qual engloba diferentes significados. Em geral, busca a explicação do que é considerada uma vida satisfatória. Vida essa que proporcione bem estar ao sujeito que a vive, produzindo estados de felicidade. A Qualidade de Vida tem se tornado um conceito que, frequentemente, se refere à evolução do processo saúde-doença. Atualmente o conceito de saúde não está associado somente à ausência de doença; relacionando-se mais com o 
estatuto de bem-estar e padrão de vida, num âmbito não apenas biológico e individual, mas também social, histórico e cultural ${ }^{16}$.

Pesquisa conduzida por Sampalis e colegas $^{17}$ teve como finalidade conhecer o impacto do tempo de espera na qualidade de vida em pacientes aguardando para cirurgia de ponte de safena. Os participantes foram avaliados pelo questionário genérico SF-36 no início, antes e após 6 meses de cirurgia. Assim, os autores constataram que não há diferenças entre os grupos na avaliação inicial. Porém, no período pré-cirurgia e após os 6 meses, o grupo que aguardou mais tempo apresentou uma redução da qualidade de vida, comparado ao grupo que aguardou por um período reduzido. Também foi observado um aumento no número de intercorrências no período pós-operatório no grupo que aguardou maior tempo de cirurgia. Portanto, os autores concluíram que a redução do tempo de espera aumenta a qualidade de vida desses pacientes ${ }^{17}$.

Estudos ${ }^{18,19}$ que visaram avaliar e comparar a qualidade de vida em portadores de doença arterial coronariana submetidos a um dos três tratamentos (clínico, cirúrgico ou por angioplastia) constataram que os sujeitos submetidos ao tratamento cirúrgico mostraram evolução mais favorável, com os homens apresentaram melhor qualidade de vida. Nesse sentido, Nogueira e colegas $^{20}$, ao avaliarem a qualidade de vida em pacientes submetidos à cirurgia de revascularização do miocárdio com e sem circulação extracorpórea, observaram melhora progressiva da qualidade de vida e retorno precoce ao trabalho, independentemente da técnica cirúrgica empregada.

Desse modo, os estudos supracitados indicam uma melhor Qualidade de Vida de pacientes portadores de cardiopatia após a cirurgia cardíaca. No presente estudo, vale ressaltar que, devido ao acesso aos pacientes e à logística da instituição, os questionários, inclusive o de Qualidade de Vida SF-12, foram aplicados no período de internação na enfermaria (entre três e dez dias após a cirurgia). Essa variável não controlada é considerada, pelos pesquisadores, uma das limitações da pesquisa, visto que tal fato pode interferir na percepção da qualidade da vida do entrevistado.

Além disso, há capacidades emocionais que permitem o enfrentamento de situações adversas e estressoras, como em uma intervenção cirúrgica.
A capacidade de resiliência, que pode ser entendida como a flexibilidade para responder às novas demandas situacionais adversas e adaptar-se a elas sem que isso cause maiores danos, exerce relevante papel na forma como o sujeito encara uma doença crônica. Nesse cenário, a resiliência seria a capacidade de o sujeito lidar com a doença e suas limitações, aderir ao tratamento proposto e readaptar-se de forma positiva ${ }^{21}$.

Nessa direção, Grings e Vigueras ${ }^{22}$, ao avaliarem a capacidade de resiliência e depressão em uma população de pacientes portadores de coronariopatias, revelam que $60,8 \%$ de sua amostra evidencia elevado índice de resiliência e 72,5\% possuem escore mínimo para depressão. Tal estudo encontrou um nível médio de resiliência de 147,36 (dp $\pm 15,18$ ). Tal dado vai ao encontro dos achados do presente trabalho, em que a média da capacidade de resiliência foi de $131( \pm 31)$.

Meister e colegas ${ }^{23}$ verificaram que pacientes com infarto agudo do miocárdio (IAM) com maior capacidade de resiliência demonstraram menos sintomas de estresse agudo após um episódio de IAM. Assim, os autores relacionam a baixa capacidade de resiliência a potenciais sintomas de estresse agudo.

Embora a atual pesquisa fora realizada com pacientes cirúrgicos, pode-se considerar a cirurgia cardíaca, bem como o IAM, uma situação de crise e, portanto, potencialmente traumática. Logo, tendo por base os achados dessa pesquisa, bem como a revisão de literatura realizada, defende-se a hipótese de que as características de resiliência de um sujeito, ou seja, a maneira como ele enfrenta as situações de crise em sua vida, relaciona-se à sua percepção de qualidade de vida. Por fim, no que diz respeito às limitações do estudo, destaca-se o tamanho da amostra que restringiu a realização de correlações mais consistentes.

\section{CONSIDERAÇÕES FINAIS}

O presente estudo visou verificar e comparar dois construtos empregados no campo da saúde: Resiliência e Qualidade de Vida. Pôde-se contatar uma correlação direta fraca entre tais conceitos. Esse achado sugere a existência de uma tendência a uma melhor qualidade de vida no que tange a aspectos físicos em indivíduos que apresentam maiores características de resiliência. 
Nesse sentido, cabe salientar a promoção de ações de cuidado da equipe de saúde no intuito de minimizar os fatores de risco e de potencializar os fatores protetivos de pacientes candidatos à cirurgia cardíaca. Essas ações visam propiciar uma melhor Qualidade de Vida após o procedimento cirúrgico. Faz-se, assim, de extrema importância conhecer os efeitos psicológicos da cirurgia cardíaca na vida desses pacientes. Por outro lado, não foi pretensão dessa investigação esgotar a temática apresentada, necessitando, assim, de estudos adicionais sobre essa população estudada.

\section{REFERÊNCIAS}

1. Rocha L, Maia TF, Silva LF. Diagnósticos de enfermagem em pacientes submetidos a cirurgias cardíaca. Acta Paul Enferm 2006; 59(3):321-326.

2. Tarasoutchi F, Montera MW, Grinberg M, Barbosa MR, Piñeiro DJ, Sánchez CRM, Barbosa MM, Barbosa GV et al. Diretriz Brasileira de Valvopatias - SBC 2011 / I Diretriz Interamericana de Valvopatias - SIAC 2011. Arq Bras Cardiol 2011; 97(5 supl. 1): 1-67

3. Minayo MC. Qualidade de vida e saúde: um debate necessário. Ciência \& Saúde Coletiva 2000; 5(1):7-18.

4. Alvares FBL. Qualidade de Vida em Crianças Portadoras de Cardiopatia Congênita [Dissertação de Mestrado]. Campo Grande (MS): Universidade Católica Dom Bosco; 2012.

5. Minayo MdS, Hartz Z, Buss PM. Qualidade de vida e saúde: um debate necessário. Ciência saúde coletiva 2000;5(1):7-18

6. Noronha MGRCS, Cardoso PS, Moraes TNP, Centa ML. Resiliência: nova perspectiva na promoção da saúde da família? Ciência e Saúde Coletiva 2009;14(2):497-506.

7. Castro EK, Moreno-Jiménez B. Resiliencia en niños enfermos crônicos: aspectos teóricos. Psicologia em Estudo 2007;12: 81-86.

8. Pesce R. et all. Adaptação transcultural, confiabilidade e validade da escala de resiliência. Cad. Saúde Pública 2005;21(2):436-448.

9. Rutter M. Resilience: Some conceptual considerations. Journal of Adolescent health 1993;14:626-631.

10. Yunes MAM, Szymanski H. Resiliência: Noção, conceitos afins e considerações críticas. In: Tavares, organizador. Resiliência e Educação. São Paulo: Cortez; 2001. P. 13-42.

11. Morais, NA, Koller, SH. Abordagem ecológica do desenvlvimento humano, psicologia positiva e resiliência:
Ênfase na saúde. In: Koller SH, organizador. Ecologia do desenvolvimento humano: Pesquisas e Intervenção no Brasil. São Paulo: Casa do Psicólogo; 2004. P. 91-107.

12. Santana JJRA, Fernandes LFB, Zanin CR, Waeteman CM, Soares M. Grupo educativo de cirurgia cardíaca em um hospital universitário: impacto psicológico. Estudos de Psicologia 2010; 27(1):31-39.

13. Brasil. Ministério da Saúde. Saúde Brasil 2009: uma análise da situação de saúde e da agenda nacional e internacional de prioridades em saúde. Brasília: Ministério da Saúde, 2009.

14. Polanczyk, CA. Fatores de risco cardiovascular no Brasil: os próximos 50 anos! Arquivos Brasileiros de Cardiologia 2005; 84(3):199-201.

15. Gus I, Fischmann A, Medina C. Prevalência dos fatores de risco da doença arterial coronariana no Estado do Rio Grande do Sul. Arquivos Brasileiros de Cardiologia 2002; 78(5):478-483.

16. Amorim LdFP. Prevalência das cardiopatias congênitas diagnosticadas ao nascimento no Hospital das Clínicas da Universidade Federal de Minas Gerais [Dissertação de Mestrado]. Belo Horizonte (MG): Universidade Federal de Minas Gerais; 2007.

17. Sampalis J, Boukas S, Liberman M, Reid T, Dupuis G. Impact of waiting time on the quality of life of patients awaiting coronary artery bypass grafting. MAJ 2001;165:429-33.

18. Favarato, MECS, Favarato D, Hueb WA, Aldrighi JM. Qualidade de vida em portadores de doença arterial coronária: comparação entre gêneros. Rev Assoc Med Bras 2006; 52(4):236-41.

19. Takiuti ME, Hueb W, Hiscock SB, Nogueira CRSdaR, Girardi P, Fernandes $\mathrm{F}$ et al . Qualidade de vida após revascularização cirúrgica do miocárdio, angioplastia ou tratamento clínico. Arquivos Brasileiros de Cardiologia 2007; 88( 5 ): 537-544.

20. Nogueira CRSR, Hueb W, Takiuti ME, Girard PBMA, Nakano T, Fernandes F et al. Qualidade de vida após revascularização cirúrgica do miocárdio com e sem circulação extracorpórea. Arquivos Brasileiros de Cardiologia 2008; 91(4):238-44.

21. Bianchini DCS, Dell'Aglio DD. Processos de resiliência no contexto de hospitalização: um estudo de caso. Paideia 2006; 16(35):427-36.

22. Grings CO, Vigueras ESR. Superando as adversidades: resiliência diante da doença arterial coronariana. Revista Brasileira de Ciências da Saúde 2012;10(32):55-61.

23. Meister RE, Weber T, Princip M, Schnyder U, Barth J, Znoj H, Schmid JP, Känel R. Resilience as a correlate of acuate stress disorder symptoms in patients with acuate myocardial infarction. Opem Heart 2015; 2(7):116-28. 